placebo as an adjuvant treatment of renal cell carcinoma: results from the phase III, randomized ATLAS trial. Annals of oncology : official journal of the European Society for Medical Oncology 2018;29:2371-8.

4. Eisen T, Frangou E, Oza B, et al. Adjuvant Sorafenib for Renal Cell Carcinoma at Intermediate or High Risk of Relapse: Results From the SORCE Randomized Phase III Intergroup Trial. 2020;38:4064-75.

5. Ravaud A, Motzer RJ, Pandha HS, et al. Adjuvant Sunitinib in High-Risk Renal-Cell Carcinoma after Nephrectomy. The New England journal of medicine 2016;375:2246-54.

6. Motzer RJ, Ravaud A, Patard JJ, et al. Adjuvant Sunitinib for High-risk Renal Cell Carcinoma After Nephrectomy: Subgroup Analyses and Updated Overall Survival Results. Eur Urol 2018;73:62-8.

7. Armstrong AJ, Halabi S, Eisen T, et al. Everolimus versus sunitinib for patients with metastatic non-clear cell renal cell carcinoma (ASPEN): a multicentre, open-label, randomised phase 2

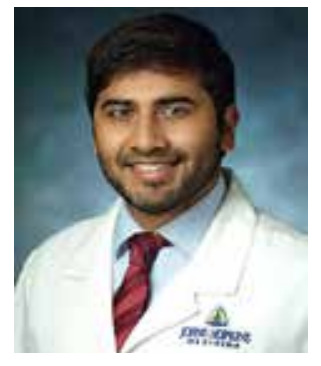

\title{
Novel Strategies in Locally Advanced Kidney Cancer - Highlights from KEYNOTE-564
}

\author{
Nirmish Singla, MD, MSCS
}

Departments of Urology and Oncology, The James Buchanan Brady Urological Institute, Johns Hopkins University School of Medicine, Baltimore, MD, USA

\section{https://doi.org/10.52733/KCJ19n2-s1-a15}

$\mathrm{W}$ ith upwards of $30 \%$ recurrence rates in patients with locally advanced kidney cancer undergoing curative-intent surgical resection, there is a critical need to develop multimodal strategies to improve outcomes in these patients. The first pre-specified interim results from the phase III KEYNOTE-564 study were presented at the 2021 ASCO Annual Meeting as an encouraging approach to address this need. In particular, the multicenter trial randomized 994 selected high-risk patients with clear cell renal cell carcinoma (ccRCC) to receive either adjuvant pembrolizumab for up to 17 cycles or placebo, with the primary endpoint of disease-free survival (DFS). Over a median follow-up of 24 months, the use of adjuvant pembrolizumab significantly improved DFS (77.3\% vs. 68.1\%; HR o.68, $\mathrm{p}=0.0010$ ). Nevertheless, longer follow-up will be required to assess the sustainability of the observed delta in DFS at 2 years. Overall survival (OS) data, a key secondary endpoint, are also still premature, with $96.6 \%$ of patients in the pembrolizumab group and $93.5 \%$ of the placebo group alive at 24 months. Adverse events were in alignment with the expected side effect profiles of immune checkpoint inhibitors (ICI).
Taken together, the findings from KEYNOTE-564 are indeed encouraging and represent, perhaps, the first positive phase III study with the adjuvant administration of an ICI for locally advanced ccRCC. Notably, data from the earlier S-TRAC trial had likewise shown improvements in DFS with the adjuvant use of sunitinib in these higher risk patients, though without demonstrable improvement in OS-thereby limiting its widespread use in this setting. Thus, we eagerly await the mature OS data to emerge from KEYNOTE-564 in order to determine whether the observed DFS benefit from adjuvant pembrolizumab will translate into improved OS for these patients or exhibit a trend similar to that of sunitinib. Furthermore, patient selection for this strategy and applicability to the non-ccRCC population remain unanswered questions. While KEYNOTE-564 explores use of ICI in the adjuvant setting, other parallel studies, including PROSPER-RCC, will help define whether the timing of perioperative ICI administration (i.e., to include neoadjuvant use while the tumor remains in situ) will play an important role in improving outcomes compared to the current standard of care. 